“一步法”合成新型不对称三甲川吲哚菁荧光染料及苂光标记

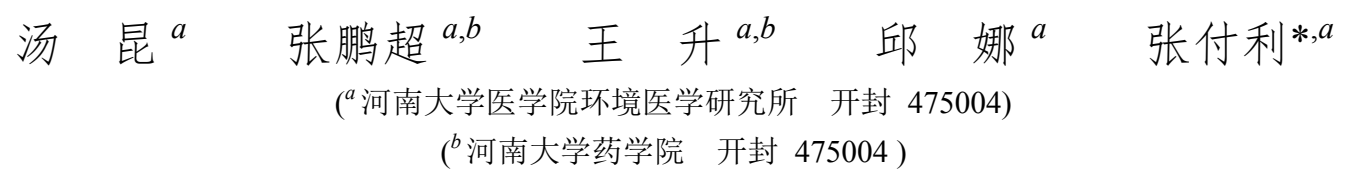

\begin{abstract}
摘要 首次合成了一种含非离子水溶性基团的水溶性不对称三甲川吲哚菁苂光染料. 以 $N-(3,5-$ 二(2-(2-甲氧乙氧基)乙 氧基)乙氧基)苄基-2,3,3-三甲基-5-磺酸基- $3 H$-吲哚、 $N$-对羧苄基-2,3,3-三甲基-5-磺酸基- $3 H$-吲哚和缩合剂 $N, N^{\prime}$-二苯基 二甲脒为原料, 采用 “一步法” 合成, 用自制简易 C18 反相硅胶填料柱分离即可得到纯品, 目标染料经 HRMS、NMR 表征; 测试了染料的紫外光谱性能、苂光光谱性能和光稳定性能; 研究了染料标记牛血清白蛋白(BSA)和细胞染色. 结 果表明: 在吲哚“ $\mathrm{N}$ ”原子上引入具有 PEG 链的非离子水溶性基团, 使目标染料合成简便, 纯化简单, 产率达到 $73 \%$; 苂 光量子产率 $(\Phi)$ 达到 0.3 ; 标记蛋白质标示率 $(\mathrm{D} / \mathrm{P})$ 为 1.87 ; 染料对固定细胞和活细胞染色结果有显著差异, 能有效区分 细胞存活状态.
\end{abstract}

关键词 三甲川吲哚菁染料; 非离子水溶性基团; 荧光量子产率; 蛋白质标记; 细胞染色

\title{
One-Step Synthesis of Novel Asymmetric Trimethine Indocyanine Fluorescent Dye and Fluorescent Labeling
}

\author{
Tang, Kun ${ }^{a}$ Zhang, Pengchao ${ }^{a, b} \quad$ Wang, Sheng ${ }^{a, b} \quad$ Qiu, $\mathrm{Na}^{a} \quad$ Zhang, Fuli ${ }^{*, a}$ \\ $\left({ }^{a}\right.$ Research Institute of Environmental Medicine, Medical College of Henan University, Kaifeng 475004) \\ ( ${ }^{b}$ Pharmaceutical College of Henan University, Kaifeng 475004)
}

\begin{abstract}
A novel water-soluble asymmetric trimethine cyanine dye which contains one nonionic hydrophilic group was first synthesized, which was prepared from one-step synthesis with $N$-carboxybenzyl-2,3,3-trimethyl-3H-indol-5-sulfonic acid, $N, N^{\prime}$-diphenylformamidine and 2,3,3-trimethyl-3H-indol-5-sulfonic acid with PEG. The pure title compound could be obtained by using the self-made $\mathrm{C} 18$ reversed-phase chromatographic column. The structure of the product was identified by NMR and HRMS. The spectra character and photostability of this dye were detected, protein labeling of albumin bovine serum (BSA) and cell stain with this dye were investigated. The results showed that the yield of the target compound could reach $73 \%$ by simple synthesis and purification, the fluorescence quantum yield was 0.3 , the $\mathrm{D} / \mathrm{P}$ of protein labeling was 1.87 . The dye could effectively distinguish between the fixed cells and living cells, because of the significant differences in the staining results.
\end{abstract}

Keywords trimethine indocyanine; nonionic hydrophilic group; fluorescence quantum yield; protein labeling; cell stain

随着半导体二极管技术的发展, 苂光标示法已逐步 代替具有放射性危害的同位素标示法, 成为生物医药领 域中具有里程碑意义的检测方法 ${ }^{[1]}$. 花菁染料是为数不 多的发光在近红外区的苂光标记用染料 ${ }^{[2]}$, 尤其是近红 外水溶性三甲川吲哚菁染料, 具有光稳定性高、特异性 好、灵敏度高、分子体积小、用样量少、最大发射波长

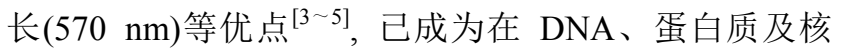
酸 ${ }^{[6]}$ 等生物分析检测中主要使用的苂光标示物. 但是,
不对称水溶性三甲川吲哚菁染料制备时，为避免大量对 称菁染料的生成, 多采用半菁法, 即 “二步法”。该方法 步骤繁琐, 分离困难, 产率较低, 大都在 $50 \%$ 以内 ${ }^{[9,10]}$. 我们设计合成的不对称三甲川吲哚菁染料, 在吲哚 “ $N$ ” 原子上引入一个含有 PEG 链的非离子水溶性基团, 可 采用 “一步法” 合成，方法简便，提纯简单，产率较高. 同时，该标示物光稳定性好、荧光强度及苂光量子产率 高，具有一定的应用开发价值. 合成路线 Scheme 1.

\footnotetext{
*E-mail: hdzf1508@163.com

Received November 12, 2015; revised January 16, 2016; published online March 11, 2016.

Project supported by the National Natural Science Foundation of China (No. 21202036).

国家自然科学基金(No. 21202036)资助项目.
} 


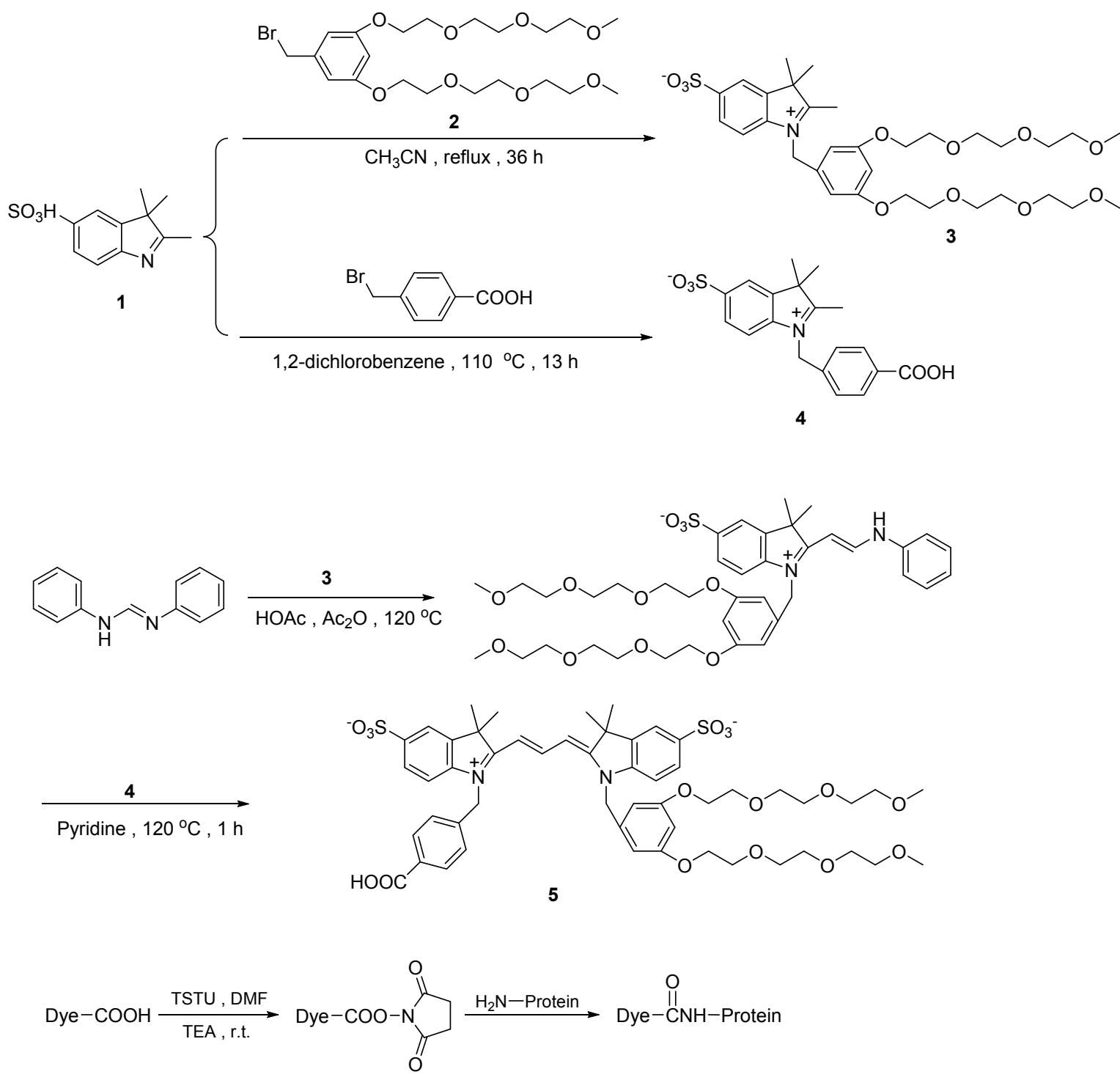

图式 1 目标染料的合成及蛋白质标记

Scheme 1 The synthetic route of the target dye and protein labeling reaction

\section{1 结果与讨论}

\section{1 化合物 3 的合成}

乙酸钾的加入起到两方面的作用. 一方面, 有机酸 钾盐提供了碱性环境, 使吲哚 “ $\mathrm{N}$ ” 成为游离状态, 提高 了 “ $\mathrm{N}$ ” 的亲核能力, 便于季铵盐的生成. 另一方面, 化 合物 $\mathbf{1}$ 的 ${ }^{1} \mathrm{H}$ NMR 中出现等宽等高的三重峰, 化学位移 $\delta 7.0 \sim 7.3$, 说明有磺酸铵盐的存在, 铵盐对目标染料活 化酯的形成有不利影响, 加入乙酸钾可使磺酸铵转化为 磺酸钾, 提高了染料与 $O$-( $N$-琥珀酰亚胺基)- $N, N, N^{\prime}, N^{\prime}$ 四甲基四氟硼酸嫝(TSTU)的酯化率, 避免了铵离子对 蛋白质和抗体标示过程中的干扰.

\section{2 目标染料 5 的合成}

我们设计染料化合物 $\mathbf{5}$ 时, 在化合物 $\mathbf{3}$ 吲哚 “ $\mathrm{N}$ ” 原子上引入了一个体积较大, 含有 $\mathrm{PEG}$ 链的非离子水
溶性基团，首先让化合物 3 和缩合剂按等物质的量充分 反应后，再加入等物质的量的化合物 4. 结果发现对称 菁染料的生成量相对较少, 不对称菁染料生成量相应增 高. 可能是因为新引入基团体积较大，增加了反应的空 间位阻, 对称菁染料的生成相对困难, 提高了不对称菁 染料的产率. 因此，化合物 5 合成时，严格控制反应物 的物质的量之比以及加料顺序，可采用 “一步法” 合成.

\section{3 目标染料的纯化}

生物标示一般在水溶液中进行, 染料水溶性对标示 有较大影响, 通常通过引入磺酸基提高菁染料的水溶 性，这样，目标产物的极性很大，主产物和副产物的极 性差别较小，分离提纯极为困难，多采用价格昂贵的制 备色谱. 我们设计合成的三甲川不对称吲哚菁染料, 由 于引入一个含有 PEG 链的非离子水溶性基团, 使目标 产物与副产物的结构和极性差别增大, 用自行设计、装 
填的简易 $\mathrm{C} 18$ 反相硅胶分离柱即可得到纯品, 产率达 $70 \%$ 以上.

\section{4 目标染料紫外-可见吸收光谱和荧光光谱性能}

配制染料浓度为 $1 \times 10^{-4} \mathrm{~mol} / \mathrm{L}$ 的 $\mathrm{H}_{2} \mathrm{O} 、 \mathrm{MeOH}$ 和 DMF 溶液, 分别测定紫外-可见吸收光谱和荧光光谱, 参照文献[11]的方法计算其苂光量子产率. 表 1 列出了 染料在不同溶剂中的光谱性质, 图 1 为染料在不同溶剂 中的紫外吸收光谱, 图 2 为染料在不同溶剂中的苂光发 射光谱.

表 1 目标染料在不同溶剂中的紫外吸收光谱和苂光光谱性 质

Table 1 UV-Vis absorption and emission spectra properties of the target dye in different solvents

\begin{tabular}{cccccc}
\hline Solvent & $\lambda_{\mathrm{ab}} / \mathrm{nm}$ & $\lambda_{\mathrm{em}} / \mathrm{nm}$ & $\begin{array}{c}\text { Stoke } \\
\text { Shift } / \mathrm{nm}\end{array}$ & $\begin{array}{c}\varepsilon / \\
\left(\mathrm{L} \cdot \mathrm{mol}^{-1} \cdot \mathrm{cm}^{-1}\right)\end{array}$ & $\Phi$ \\
\hline $\mathrm{H}_{2} \mathrm{O}$ & 559 & 573 & 14 & 87368 & 0.361 \\
$\mathrm{MeOH}$ & 565 & 577 & 12 & 138440 & 0.399 \\
$\mathrm{DMF}$ & 576 & 589 & 13 & 71080 & 0.399 \\
\hline
\end{tabular}

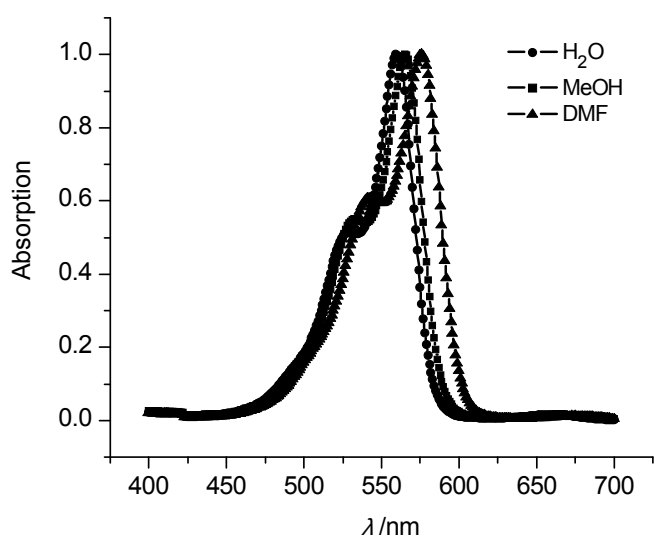

图 1 目标染料在 $\mathrm{H}_{2} \mathrm{O} 、 \mathrm{MeOH}$ 和 DMF 中的紫外吸收光谱 Figure 1 Absorption spectra of the target dye in $\mathrm{H}_{2} \mathrm{O} 、 \mathrm{MeOH}$ and DMF

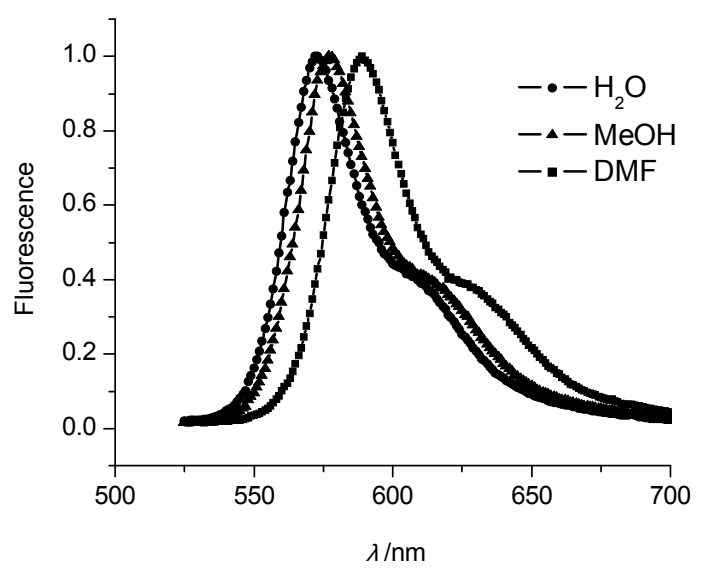

图 2 目标染料在 $\mathrm{H}_{2} \mathrm{O} 、 \mathrm{MeOH}$ 和 DMF 中的荧光发射光谱

Figure 2 Fluorescence emission spectra of the target dye in $\mathrm{H}_{2} \mathrm{O}, \mathrm{MeOH}$ and DMF
可以看出，染料在不同溶剂中的最大吸收波长和最 大发射波长均在近红外区; 在质子溶剂中的最大吸收和 最大发射波长较非质子溶剂中明显变小，发生了蓝移， 可能的原因是质子溶剂对分子的溶剂效应增强, 不利于 分子发生聚集.

染料的苂光量子产率随着溶剂的极性增加而降低, 但该染料在水中的荧光量子产率在 0.3 以上, 高于一般 的参比染料. 原因可能是因为目标化合物中, 在吲哚 “ $\mathrm{N}$ ” 原子上引入了一个非离子水溶性基团，醚链上的 “ $\mathrm{O}$ ”易与水形成分子间氢键，从而增加染料的水溶性， 降低了染料在水中自聚而导致的荧光自淬灭，提高了荧 光量子产率 ${ }^{[12]}$.

\section{5 目标染料的光稳定性}

将染料配成 $1 \times 10^{-5} \mathrm{~mol} / \mathrm{L}$ 的 $\mathrm{H}_{2} \mathrm{O}$ 溶液, 在碘铇灯照 射下, 每隔 $2 \mathrm{~h}$ 测一次吸光度. 以相对吸光度为纵坐标, 照射时间为横坐标作染料光降解曲线.

染料在水中的光稳定性如图 3 所示, 摩尔吸光度随 光照时间延长几乎没有变化, 说明该目标化合物光稳定 性高，溶液光谱性质稳定，有利于长时间保存，便于生 物标记时使用.

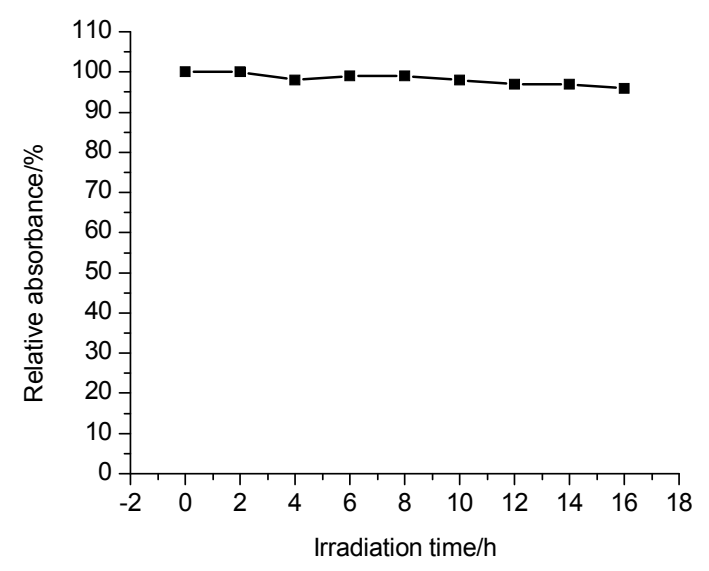

图 3 目标染料在水中的光稳定性

Figure 3 Photostability of the target dye in water

\section{6 牛血清白蛋白(BSA)的苂光标记}

在生物标记中，标记蛋白质最适合应用的 $\mathrm{D} / \mathrm{P}$ 值是 $1 \sim 2^{[13]}$. 标记结果表明, 目标染料与 BSA 的物质的量之 比为 $4: 1$ 时, D/P 值达 1.87. 可见, 该标示物对蛋白质有 较高的标记率.

\section{7 细胞成像}

目标染料对固定细胞的染色结果见图 4(a), 对活细 胞的染色结果见图 4(b). 染料与固定细胞共孵育 $30 \mathrm{~min}$, 对比同一视野明场图像(图 4a1), 苂光显微镜下, 可以看 到整个细胞被染色，细胞核苂光亮度最为明显(图 4a2). 
这可能因为细胞经多聚甲醛固定后, 细胞膜上的蛋白质 固缩, 细胞膜通透性增加, 目标染料进入细胞内的量增 多; 目标染料结构中含枝状 PEG 醚链, 使染料水溶性增 加的同时, 又具有一定的亲酯性, 在很大程度上增强了 与细胞膜的亲和力, 进而增加了对细胞的透过能力; 细 胞核内含有大量核酸和蛋白质, 核酸和蛋白质上的活性 基团结合目标染料的量增多 ${ }^{[14]}$, 引起固定细胞核苂光 强度最明显.

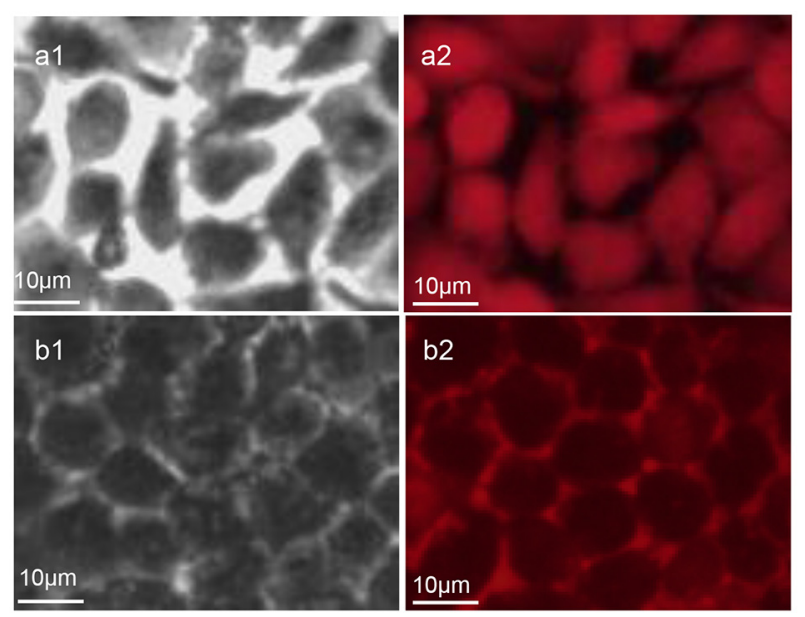

图 4 目标染料与人肝癌 SMMC-7721 细胞的固定细胞成像(a) 和活细胞成像(b)

Figure 4 The fluorescence images of fixed (a) and viable (b) SMMC-7721 cells stained by the target dye

a1 and b1 for bright-field, a2 and b2 for fluorescence

该目标染料与活细胞共捊育 $30 \mathrm{~min}$, 对照同一视野 明场图像(图 4b1), 苂光显微镜下可见仅有少量染料透 过细胞膜进入细胞内部, 其余大部分染料聚集在细胞膜 表面, 能清晰观察到细胞外周轮廓(图 4b2). 这可能是因 为活细胞膜生理结构和功能完整, 细胞膜上的通道和载 体不允许目标染料自由通过.

研究说明, 染料能对固定细胞和活细胞分别染色, 染色效果有显著差异, 能有效区分细胞存活状态, 在活 细胞成像和监测中晚期凋亡细胞中具有一定的应用价 值.

\section{2 结论}

本文采用 “一步法” 合成了一种新型水溶性不对称 三甲川吲哚菁荧光标示物, 用自制填装的 C18 反相硅胶 柱分离可得到纯品; 目标染料的合成工艺简单, 分离简 便, 重复性好, 产率较高; 目标染料荧光量子产率高, 摩尔消光系数大, 具有良好的水溶性, 光稳定性好, 标 示蛋白质方法简便, 标示率高, 可用于细胞染色, 有效 区分细胞存活状态, 是一种性能优良的新型荧光标示 物，在生物标示领域有很高的开发应用价值.

\section{3 实验部分}

\section{1 试剂与仪器}

MICROTOF-QII 电喷雾四级杆飞行时间质谱仪(德 国 BRUKER 公司); AVANCE-400 MHz 核磁共振仪(瑞 士布鲁克公司); PE-Lambda35 双通道紫外-可见分光光 度计(美国珀金埃尔默仪器责任有限公司); FluoroSENS 稳态苂光光谱仪(英国 Gilden 公司); 苂光显微镜(Leica DM2500).

对甲苯磺酰氯(AR，阿拉丁试剂); 三乙二醇单甲醚 (AR，东京化成工业株式会社); 4-磺酸基苯肼(AR，华联 化工实业有限公司)；3-甲基-2-丁酮(AR，萨恩化学技术 有限公司); 原甲酸三乙酯(AR，阿拉丁试剂); 罗丹明 $\mathrm{B}$ (GR，梯希爱化成工业发展有限公司); TSTU (AR，Alfa Aesar 试剂); 三乙胺(AR, Alfa Aesar 试剂); C18 反相柱 填料(200 300 目，西安交大保赛生物技术股份有限公 司); 人肝癌细胞 SMMC-7721(来自中国医学科学院基 础医学研究所); 去离子水为二次蒸馏水; 其它试剂均 为分析纯

\section{2 实验步骤}

3.2.1 N-(3,5-二(2-(2-甲氧乙氧基)乙氧基)乙氧基)芐 基-2,3,3-三甲基-5-磺酸基-3H-吲哚(3)的合成

化合物 1 参照文献[15]合成，化合物 2 参照文献[16] 合成.

将化合物 1 (1.0 g, $4.18 \mathrm{mmol})$ 、乙酸钾 $(0.45 \mathrm{~g}, 4.59$ $\mathrm{mmol})$ 和甲醇 $(30 \mathrm{~mL}$ )加入到 $100 \mathrm{~mL}$ 三颈圆底烧瓶中, 室温反应 $0.5 \mathrm{~h}$, 蒸出甲醇, 再加入化合物 2 (3.1 g, 6.26 $\mathrm{mmol})$ 和乙腈 $(30 \mathrm{~mL})$, 氩气保护下回流反应 $36 \mathrm{~h}$, 反应 结束后旋蒸出乙腈，粗产品用正相硅胶填料柱分离 $[V$ (甲醇 $): V($ 二氯甲烷 $)=1: 2$ ]得红色固体 $1.7 \mathrm{~g} \mathrm{3}^{[11]}$, 产率 $61 \%$. m.p. $>300{ }^{\circ} \mathrm{C} ;{ }^{1} \mathrm{H}$ NMR (DMSO- $\left.d_{6}, 400 \mathrm{MHz}\right)$ $\delta: 7.40(\mathrm{~d}, J=1.6 \mathrm{~Hz}, 1 \mathrm{H}, 4-\mathrm{H}), 7.34$ (dd, $J=1.6,8.4 \mathrm{~Hz}$, $1 \mathrm{H}, 6-\mathrm{H}), 6.55$ (d, $J=8.4 \mathrm{~Hz}, 1 \mathrm{H}, 7-\mathrm{H}), 6.38(\mathrm{t}, J=2.0 \mathrm{~Hz}$, $1 \mathrm{H}, \mathrm{OArH}), 6.35$ (d, J=2.0 Hz, 2H, OArH), 4.68 (s, 2H, $\mathrm{ArCH}_{2}$ ), 3.99 (t, $\left.J=4.8 \mathrm{~Hz}, 4 \mathrm{H}, \mathrm{ArOCH}_{2}\right), 3.68$ (t, $J=4.8$ $\left.\mathrm{Hz}, 4 \mathrm{H}, \mathrm{ArOCH}_{2} \mathrm{CH}_{2}\right), 3.59 \sim 3.39\left(\mathrm{~m}, 16 \mathrm{H}, \mathrm{OC}_{2} \mathrm{H}_{4} \mathrm{O}-\right.$ $\left.\mathrm{C}_{2} \mathrm{H}_{4} \mathrm{O}\right), 3.34\left(\mathrm{~s}, 3 \mathrm{H}, \mathrm{N}=\mathrm{CCH}_{3}\right), 3.22\left(\mathrm{~s}, 6 \mathrm{H}, \mathrm{OCH}_{3}\right), 1.32$ $\left(\mathrm{s}, 6 \mathrm{H}, \mathrm{CH}_{3}\right)$; $\mathrm{MS} m / z: 654.3[\mathrm{M}+\mathrm{H}]^{+}$.

3.2.2 $\mathrm{N}$-对羧芐基-2,3,3-三甲基-5-磺酸基-3H-吲哚 (4) 的合成

将化合物 1 ( $1.0 \mathrm{~g}, 4.18 \mathrm{mmol})$ 、乙酸钾 $(0.45 \mathrm{~g}, 4.59$ $\mathrm{mmol}$ )和甲醇 $(30 \mathrm{~mL}$ )加入到 $100 \mathrm{~mL}$ 三颈圆底烧瓶中, 室温反应 $0.5 \mathrm{~h}$, 蒸出甲醇，再加入对溴甲基苯甲酸(1.0 $\mathrm{g}, 4.65 \mathrm{mmol})$ 和邻二氯苯 $(10 \mathrm{~mL})$, 氩气保护下 $110{ }^{\circ} \mathrm{C}$ 反 应 $4 \mathrm{~h}$, 反应结束后加入甲醇 $(50 \mathrm{~mL})$, 超声析出粉红色 
固体 $1.0 \mathrm{~g} \mathrm{4}^{[9]}$, 产率 $66 \%$. m.p. $>300{ }^{\circ} \mathrm{C} ;{ }^{1} \mathrm{H}$ NMR (DMSO- $\left.d_{6}, 400 \mathrm{MHz}\right) \delta: 8.08(\mathrm{~d}, J=1.6 \mathrm{~Hz}, 1 \mathrm{H}, 4-\mathrm{H})$, 7.96 (d, $J=8.0 \mathrm{~Hz}, 2 \mathrm{H}, \mathrm{OOCArH}), 7.75$ (dd, $J=1.6,8.4$ $\mathrm{Hz}, 1 \mathrm{H}, 6-\mathrm{H}), 7.70$ (d, $J=8.4 \mathrm{~Hz}, 1 \mathrm{H}, 7-\mathrm{H}), 7.48$ (d, $J=$ $8.0 \mathrm{~Hz}, 2 \mathrm{H}, \mathrm{OOCArH}), 5.91$ (s, 2H, Ar- $\left.\mathrm{CH}_{2}\right), 2.94$ (s, 3H, $\left.\mathrm{N}=\mathrm{C}-\mathrm{CH}_{3}\right), 1.62\left(\mathrm{~s}, 6 \mathrm{H}, \mathrm{CH}_{3}\right)$.

\subsection{3 目标染料的合成}

缩合剂 $N, N^{\prime}$-二苯基甲脒参照文献[17]合成.

将化合物 $3(500 \mathrm{mg}, 0.765 \mathrm{mmol}) 、 N, N^{\prime}$-二苯基二甲 脒 $(150 \mathrm{mg}, 0.765 \mathrm{mmol}) 、 乙$ 酸 $(3 \mathrm{~mL})$ 和乙酸䣶 $(3 \mathrm{~mL})$ 加 入到 $50 \mathrm{~mL}$ 三颈圆底烧瓶中, 氩气保护下 $120{ }^{\circ} \mathrm{C}$ 反应约 $1 \mathrm{~h}, \mathrm{TLC}$ [ $V$ (甲醇) $: V$ (二氯甲烷 $)=1: 2$ ] 跟踪, 每隔 20 $\min$ 取样点板, 黄色原料点消失后, 加入化合物 4 (286 $\mathrm{mg}, 0.765 \mathrm{mmol})$ 和吡啶 $(0.5 \mathrm{~mL})$, 氩气保护下 $120{ }^{\circ} \mathrm{C}$ 继 续反应 $1 \mathrm{~h}$, 反应结束后冷却至室温, 加入乙酸乙酯析 出固体, 抽滤, 用自制 $\mathrm{C} 18$ 反相硅胶填料柱分离 $[V($ 甲 醇) $: V($ 乙腈 $)=1: 5]$, 分为三个色带, 接收第二个色带, 得到红棕色固体 $579 \mathrm{mg}$, 产率 73\%. m.p. $>300{ }^{\circ} \mathrm{C} ;{ }^{1} \mathrm{H}$ NMR (DMSO- $\left.d_{6}, 400 \mathrm{MHz}\right) \delta: 9.89$ (d, $J=8.4 \mathrm{~Hz}, 1 \mathrm{H}, \beta$ protonof the bridge), $7.95\left(\mathrm{~d}, J=1.6 \mathrm{~Hz}, 2 \mathrm{H}, 4,4^{\prime}-\mathrm{H}\right), 7.91$ (d, $J=8.0 \mathrm{~Hz}, 2 \mathrm{H}$, OOCArH), $7.50(\mathrm{~d}, J=8.4 \mathrm{~Hz}, 1 \mathrm{H}$, 6-H), $7.28\left(\mathrm{~d}, J=8.4 \mathrm{~Hz}, 2 \mathrm{H}, 7,7^{\prime}-\mathrm{H}\right), 7.22(\mathrm{~d}, J=8.0 \mathrm{~Hz}$, $2 \mathrm{H}, \mathrm{OOCArH}), 7.16(\mathrm{~d}, J=8.4 \mathrm{~Hz}, 2 \mathrm{H}, \alpha$ proton of the bridge), 7.00 (d, $\left.J=8.4 \mathrm{~Hz}, 1 \mathrm{H}, 6^{\prime}-\mathrm{H}\right), 6.55 \sim 6.48(\mathrm{~m}, 3 \mathrm{H}$, $\mathrm{OArH}), \quad 5.49 \quad\left(\mathrm{~s}, \quad 2 \mathrm{H}, \quad \mathrm{CH}_{2} \mathrm{ArCOOH}\right), \quad 5.09 \quad(\mathrm{~s}, \quad 2 \mathrm{H}$, $\mathrm{CH}_{2}$-Ar-O), 4.02 (t, $J=4.8 \mathrm{~Hz}, 4 \mathrm{H}, \mathrm{ArOCH}_{2}$ ), 3.67 (t, $J=$ $\left.4.8 \mathrm{~Hz}, \quad 4 \mathrm{H}, \quad \mathrm{ArOCH}_{2} \mathrm{CH}_{2}\right), 3.52 \sim 3.38(\mathrm{~m}, 16 \mathrm{H}$, $\left.\mathrm{OC}_{2} \mathrm{H}_{4} \mathrm{OC}_{2} \mathrm{H}_{4} \mathrm{O}\right), 3.20\left(\mathrm{~s}, 6 \mathrm{H}, \mathrm{OCH}_{3}\right), 1.65\left(\mathrm{~s}, 12 \mathrm{H}, \mathrm{CH}_{3}\right)$; ${ }^{13} \mathrm{C}$ NMR (DMSO- $\left.d_{6}, 100 \mathrm{MHz}\right) \delta: 185.92,175.25$, $172.57,160.17,146.06,143.35,143.03,142.06,138.33$, $130.07,129.87,126.36,125.93,125.55,120.11,119.77$, 111.04, 107.73, 105.09, 99.95, 99.66, 71.29, 69.91, 69.80, 69.61, 68.81, 67.29, 58.09, 47.08, 45.29, 29.17, 27.45, 15.25; HRMS (ESI) calcd for $\mathrm{C}_{52} \mathrm{H}_{65} \mathrm{~N}_{2} \mathrm{O}_{16} \mathrm{~S}_{2}[\mathrm{M}+2 \mathrm{H}]^{+}$: 1037.3776, found 1037.3771.

\subsection{4 目标染料的活化与标记牛血清白蛋白}

按物质的量之比 $1: 1.5$, 在三颈瓶中加入目标染料 和 2-琥珀酰亚胺基-1,1,3,3-四甲基腿四氟硼酸酯(TSTU), 再加入少量三乙胺和 DMF, 用锡纸包裹, 氩气保护下室 温反应 $2 \mathrm{~h}$, 反应结束后将反应液加入到乙酸乙酯中, 析出蓝色沉淀, 依次用乙酸乙酯、丙酮和乙醚洗涤, 真 空干燥, 得到染料活化酯.

将染料活化酯溶于无水无氨的 DMF 中, 配成浓度 为 $10 \mathrm{~g} / \mathrm{L}$ 的染料储备溶液. 将牛血清白蛋白(BSA)溶于 嶰酸盐缓冲溶液中 $(\mathrm{pH}=9.18)$, 配成浓度为 $56 \mathrm{~g} / \mathrm{L}$ 的蛋
白质储备溶液. 以不同物质的量之比, 将两种储备溶液 混合, 室温反应 $2 \mathrm{~h}$, 反应结束后, 用 G 25 葡聚糖凝胶柱 分离, 测定 280、567 $\mathrm{nm}$ 下标记蛋白质的吸光度, 利用 下式 ${ }^{[11]}$ 计算染料标记蛋白的 D/P 值.

$$
\frac{D}{P}=\frac{A_{\mathrm{dye}} E_{\mathrm{prot}}}{\left(A_{280}-X A_{\mathrm{dye}}\right) E_{\mathrm{dye}}}
$$

式中: $D$ 为苂光标示物的摩尔数; $P$ 为牛血清蛋白的摩尔 数; $A_{\mathrm{dye}}$ 为苂光标示物在最大吸收波长处的吸光度; $A_{\mathrm{prot}}$ 为牛血清蛋白在 $280 \mathrm{~nm}$ 处的吸光度; $E_{\mathrm{dye}}$ 为苂光标示物 的摩尔吸光系数; $E_{\text {prot }}$ 为牛血清蛋白的摩尔吸光系数; $X$ 为纠正因子: 荧光标示物在 $280 \mathrm{~nm}$ 的吸光度和荧光标 示物最大吸收波长处的吸光度之比.

\subsection{5 固定细胞和活细胞染色}

人肝癌细胞 $(S M M C-7721)$ 培养在含 $10 \%(V / V)$ 热灭 活胎牛血清的高糖 DMEM 培养基中, 于 $\mathrm{CO}_{2}$ 浓度为 $5 \%$, 温度为 $37{ }^{\circ} \mathrm{C}$ 的恒温密闭培养箱中培养, 使用对数生长 期的细胞进行实验.

以细胞浓度 $1 \times 10^{8} \cdot \mathrm{L}^{-1}$ 接种于 6 孔板底部的盖玻片 上, 于恒温培养箱中继续培养至细胞贴壁. 倾去培养液, PBS 缓冲溶液洗涤 3 次. 用 $4 \%$ 多聚甲醛 $4{ }^{\circ} \mathrm{C}$ 下固定细 胞, PBS 缓冲溶液洗涤, 加入 $2 \mu \mathrm{mol} / \mathrm{L}$ 目标染料的 PBS 溶液, 室温下捊育 $30 \mathrm{~min}, \mathrm{PBS}$ 缓冲溶液洗涤 3 次, 每次 $10 \mathrm{~min}$, 封片后置于徕卡 DM2500 苂光显微镜下, 激发 波长 $505 \mathrm{~nm}$, 采集波长 $590 \mathrm{~nm}$, 观察并拍照.

细胞接种于 6 孔板盖玻片并贴壁后, 不经多聚甲醛 固定, 在细胞存活状态下, 加浓度为 $2 \mu \mathrm{mol} / \mathrm{L}$ 目标染料 的 PBS 溶液, 浸没盖玻片. 于恒温培养箱中, $37{ }^{\circ} \mathrm{C}$ 下捊 育 $30 \mathrm{~min}, \mathrm{PBS}$ 缓冲溶液洗涤, 于徕卡 DM2500 荧光显 微镜下, 激发波长 $505 \mathrm{~nm}$, 采集波长 $590 \mathrm{~nm}$, 观察并拍 照.

辅助材料(Supporting Information) 苂光染料化合物 $\mathbf{5}$ 的表征图谱. 这些材料可以免费从本刊网站(http://siocjournal.cn/)上下载.

\section{References}

[1] Wang, K.; Liu, Z.-L.; Jiang, K. Acta Chim. Sinica 2014, 72, 590 (in Chinese).

(王魁, 刘自力, 蒋凯, 化学学报, 2014, 72, 590.)

[2] Zhang, S.-X.; Yang, X.-B.; Wen, X.-H.; Ding, S.-T.; Wang, A.-B. Chin. J. Org. Chem. 2014, 34, 223 (in Chinese).

(张守金金, 杨小兵, 温晓红, 丁松涛, 王安邦, 有机化学, 2014, 34, 223.)

[3] Chen, X.-Y.; Guo, L.; Zheng, C.-G.; Gao, H.-Y.; Wang, H.-J.; Zhang, D. Chin. J. Org. Chem. 2012, 32, 1445 (in Chinese). (陈秀英, 郭琳, 郑昌戈, 高海燕, 王海军, 张丹, 有机化学, 2012, 32, 1445.) 
[4] Gao, L.-H.; Zhang, J.-F.; Sun, Q.-L.; Wang, K.-Z. Acta Chim. Sinica 2012, 70, 441 (in Chinese).

(高丽华, 张金凤, 孙庆玲, 王科志, 化学学报, 2012, 70, 441.)

[5] Park, J. W.; Kim, Y. S.; Lee, K. J. Bioconjugate Chem. 2012, 23, 350 .

[6] Stennett, E. M. S.; Ma, N.; Vaart, A. J. Phys. Chem. B 2014, 118, 152.

[7] Kozer, N.; Barua, D.; Henderson, C.; Nice, E. C.; Burgess, A. W.; Hlavacek, W. S. Biochemistry 2014, 53, 2594.

[8] Draghici, B.; Ilies, M. A. J. Med. Chem. 2015, 58, 4091.

[9] Song, B.; Liu, H.; Yu, C.-C.; Ma, W.-H.; Peng, X.-J. Acta Chim. Sinica 2010, 68, 2569 (in Chinese).

(宋波, 刘浩, 余翠翠, 马文辉, 彭孝军, 化学学报, 2010, 68, 2569.)

[10] Song, F.-L.; Wang, L.; Qiao, X.-Q.; Wang, B.-S.; Sun, S.-G.; Fan, J.-L.; Zhang, L.-H.; Peng, X.-J. Org. Biomol. Chem. 2010, 8, 4249.

[11] Tang, K.; Qiu, N.; Wu, P.; Jia, C.-H.; Wang, S.; Zhang, F.-L. Chin. J. Appl. Chem. 2014, 31, 1256 (in Chinese).
(汤昆, 邱娜, 吴品, 贾春辉, 王升, 张付利, 应用化学, 2014, 31, 1256.)

[12] Pauli, J.; Grabolle, M.; Brehm, R. B.; Spieles, M. Bioconjugate Chem. 2011, 22, 1298.

[13] Shen, B.-F.; Chen, Z. L.; Liu, M.-P. Recombinant Antibody, Science Press, Beijing, 2005, pp. 440 441 (in Chinese).

(沈倍奋, 陈志南, 刘民培, 重组抗体, 科学出版社, 北京, 2005, p. $440 \sim 441$.)

[14] Vasilev, A.; Deligeorgiev, T.; Kaloyanova, S. Color Technol. 2010, $127,69$.

[15] Oushiki, D.; Kojima, H.; Terai, T.; Arita, M.; Hanaoka, K.; Urano, Y.; Nagano, T. J. Am. Chem. Soc. 2010, 132, 2795.

[16] Rio, Y.; Nicoud, J. F.; Rehspringer, J. L.; Nierengarten, J. F. Tetrahedron Lett. 2000, 41, 10207.

[17] Wen, J.-F.; Liu, R.-T. Sci. Technol. Eng. 2012, 12, 1185 (in Chinense).

(温俊峰, 刘日涛, 科学技术与工程, 2012, 12, 1185.) 